\title{
Simulation of superelastic NiTi wire force application on orthodontic alignment case
}

\author{
A Fathallah*, T Hassine*, S Alamri**, F Gamaoun** and M Wali** \\ *Laboratory of Mechanics of Sousse, National Engineering School of Sousse, University of Sousse, Sousse, Tunisia \\ **Department of Mechanical Engineering, College of Engineering, King Khalid University, Abha, Saudi Arabia \\ Corresponding Author: aroua.fathallah.07@gmail.com
}

Submitted: $29 / 10 / 2019$

Revised: $\quad 30 / 12 / 2020$

Accepted: 11/01/2021

\begin{abstract}
Nickel Titanium (NiTi) orthodontic wires are the most accurate initial wires to align the teeth by producing the less painful bone remodeling. Coupling their mechanical response, while they are engaged in the oral cavity, with the bone remodeling would permit to elucidate the reaction and the effect of this wire during the orthodontic tooth alignment. Therefore, A two-dimensional numerical long-term orthodontic tooth correction was performed to simulate a tooth intrusion movement. This simulation was achieved by taking into consideration the effect of an orthodontic appliance action on three multiteeth models. The superelastic behavior of the NiTi wire was integrated in the computer software ABAQUS via a $U M A T$ subroutine. The orthodontic arch was initially deformed to simulate its insertion in brackets and then unloaded to apply an orthodontic load and produce initial tooth displacement. A bone remodeling process was implemented to displace the teeth depending on the strain within the periodontal ligament. The results were obtained for a treatment period of 30 days. The NiTi wire was able to recuperate its initial position, and the teeth were aligned. The numerical results of the teeth displacement were in agreement with what is expected to be produced during the alignment phase.
\end{abstract}

Keywords: Bone remodeling; Orthodontic tooth correction; Simulation; Superelastic NiTi wire; Multi-teeth effect.

\section{INTRODUCTION}

Nickel Titanium (NiTi) shape memory alloys have become one of the most exploited smart materials. Their thermomechanical characteristics have offered the possibility to develop new technical solutions and design new products used in different fields (Mohd et al., 2014). Besides being able to recover their initial shape, these materials are characterized by their superelastic property, as their reversible deformation could reach $8 \%$ (Wagner and Schaefer, 2010). These properties are due to the occurrence of a reversible martensitic transformation. The latter is activated when the alloy is subjected to large deformation or when its temperature exceeds the temperature value inducing the martensitic transformation. Thanks to these properties, NiTi shape memory alloys have been widely used, in first stage of orthodontic tooth treatment, as orthodontic wires. Their ability to deliver light continuous and constant load over a long period of time is highly recommended for producing orthodontic tooth displacement without damaging the periodontium (Aghili et al., 2015). Indeed, when the load is greater than the optimal orthodontic force, a root resorption may develop causing tooth displacement obstruction for several days (Proffit et al., 1999).

When it is inserted in the bracket slots, the wire is released and applies a permanent orthodontic force. This load applied on the teeth crown through orthodontic bracket stimulates a biological process known as bone remodeling (Proffit et al., 1999). The bone remodeling is the mechanism that provokes tooth displacement depending on a mechanical stimulus. In fact, the compressed side of Periodontal Ligament (PDL) surrounding the tooth root leads to the destruction of the bone, whereas the under-pressure side engenders bone regeneration. This phenomenon has been used to simulate different orthodontic tooth movements. 
Some researchers simulated orthodontic tooth movements resulting from the application of a load directly on the tooth crown and trigged the bone remodeling as a function of the strains within the PDL (Marangalou et al., 2009; Wang et al., 2014; Zargham et al., 2016). Chen et al. (2014) have considered the capillary blood pressure within the PDL, as the mechanical stimulus to achieve orthodontic bone remodeling. None of the aforesaid work has considered the action of an orthodontic wire. Canales et al. (2013) simulated the activation and deactivation of a stainless-steel wire in the presence of several teeth. Their simulation had only been performed to produce initial tooth displacement. Hamanaka et al. (2017) reported a numerical three-dimension space closure using stainless-steel wire inserted in fixed bracket slots. In order to achieve initial tooth displacement, they applied a constant retraction load on the wire and a reaction force on the bracket. Then, they determined the bone remodeling rate according to the initial tooth displacement.

Moreover, during initial stage of treatment, the orthodontist usually inserts NiTi wire in the bracket slots and allows it to react by itself until it resumes its initial arch shape. Some researchers have carried out numerical bending tests to evaluate the recovery force of the wire during unloading without taking into account the bone remodeling process (Naceur et al., 2014; Elkhal Letaief et al., 2018; Razali and Mahmud, 2019) . The effect of superelastic wire deactivation has not been considered to simulate long-term orthodontic tooth correction (Gannoun et al., 2018).

Despite the huge use of the superelastic $N i T i$ wires for aligning irregular teeth, none of the aforementioned studies has, as far as we know, simulated an orthodontic tooth correction using this wire. Thus, the aim of this work is to simulate a long-term orthodontic tooth alignment using superelastic NiTi arch wire. The simulation reflects the reality of the orthodontic tooth treatment since it takes into consideration an almost real reaction of the wire when it is activated and correlates the tooth movement to a bone remodeling law. In fact, the wire is initially activated by following the irregular teeth position in the oral cavity and then unloaded to apply an orthodontic load. Furthermore, the simulation considers the action of superelastic behavior of the NiTi wire in the presence of one, three and five teeth.

In this work, the mechanical and biomechanical behaviors will be presented, and the numerical method will be discussed to elucidate the coupling procedure between the orthodontic appliance action and the bone remodeling process to simulate a tooth intrusion movement. Then, the simulation results will be analyzed such as the mechanical response of the superelastic wire on the three different teeth models, the teeth motion rate, and the deformations within PDLs.

\section{MATERIALS AND METHODS}

\section{Orthodontic appliance model}

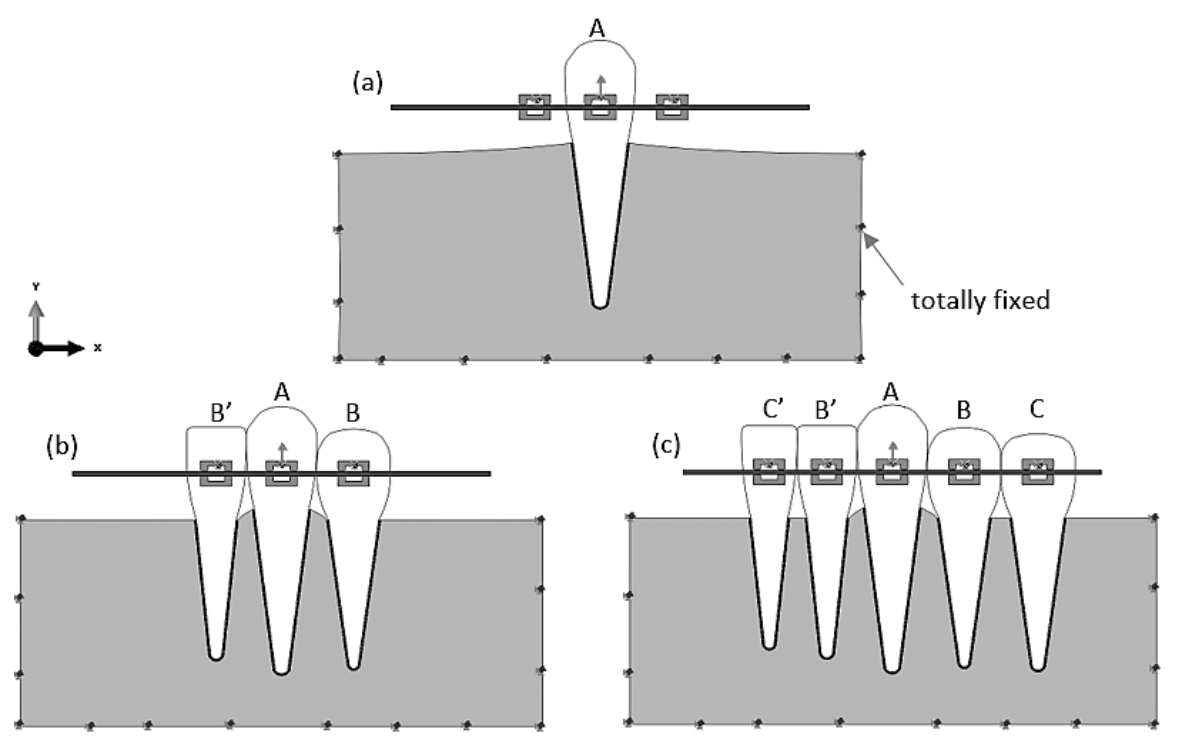

Figure 1. Boundary conditions during wire activation step in: (a) one-tooth model, (b) three-teeth model, and (c) five-teeth model. 
The orthodontic appliance consisted of three or five $0.7 \times 0.5 \mathrm{~mm}^{2}$ slot brackets made of stainless steel, in which a $0.43 \times 0.64 \mathrm{~mm}^{2}$ superelastic NiTi arch wire was inserted. The wire was, at first, created in its initial position, and the median bracket was not in contact with the tooth crown. The distance between the brackets was determined according to the teeth size. During the whole analysis, a frictional coefficient of 0.2 was assumed to simulate the interaction between arch wire and bracket slots ( Elkhal Letaief et al., 2018). According to Moore et al. (1999), the temperature at the oral cavity was equal to $308.5 \mathrm{~K}$. No boundary condition was applied on the wire to allow it to slip between the bracket slots. In the first model presented in Figure 1(a), the right and left brackets were completely constrained during the whole analysis. However, in the second and third models, the brackets were only constrained during the activation step, as displayed in Figure 1(b) and 1(c).

The superelastic behavior of the arch wire was implemented via a UMAT subroutine based on the work of Qidwai and Lagoudas (2000). The consecutive model describes the pseudoelastic behavior that took into account the occurrence of the forward and backward martensitic transformations. In this consecutive model, the Gibbs free energy is considered as thermodynamic potential with two internal state variables, which are the transformation strain and the martensitic volume fraction. The parameters required for running this consecutive model are defined in the work of Elkhal Letaief et al. (2017). These parameters are the Young's modulus of the austenite and martensitic phase $E^{A}$ and $E^{M}$, the Poisson's ratio $v^{A}$ and $v^{M}$, the coefficient of thermal expansion $\alpha$, the stress influence coefficient $\rho \Delta s_{0}$, the forward and reverse transformation temperatures, and the maximum transformation strain $H$. The values of these parameters are mentioned in Table 1.

Table 1. Thermoelastic and transformation properties of superelastic NiTi alloy (Elkhal Letaief et al., 2017).

\begin{tabular}{llllll}
\hline Parameters & Values & Units & Parameters & Values & Units \\
\hline \multicolumn{2}{l}{$\begin{array}{l}\text { Thermoelastic properties } \\
E^{A}\end{array}$} & & $v^{A}$ & 0.33 & \\
\multicolumn{1}{c}{$E^{M}$} & 11.2 & $\mathrm{GPa}$ & $v^{M}$ & 0.33 & \\
\hline \multicolumn{2}{l}{ Transformation properties } & & $\alpha$ & $2.210^{-5}$ & $\mathrm{~K}^{-1}$ \\
$\rho \Delta s_{0}$ & -0.318 & $\mathrm{MPa} \mathrm{K}{ }^{-1}$ & $A_{s}$ & & \\
$M_{s}$ & 264 & $\mathrm{~K}$ & $A_{f}$ & 262 & $\mathrm{~K}$ \\
$M_{f}$ & 241 & $\mathrm{~K}$ & $H$ & 282 & $\mathrm{~K}$ \\
\hline
\end{tabular}

\section{Biological model}

Three two-dimensional plane-strain models were constructed using ABAQUS software. One, three, and five mandibular teeth were set up in the alveolar bone as it is illustrated in Figure 1. The teeth considered in the most complete model, as exhibited from left to right in Figure 1(C), were the central incisor $C^{\prime}$, the lateral incisor $B$, the canine $A$, the first premolar $B$, and the second premolar $C$. The total teeth height, from apex to crown, and the maximum mesiodistal width are mentioned in Table 2 (Scheid et al., 2012). The thickness of PDLs, surrounding each tooth root, was $0.2 \mathrm{~mm}$. In each model, the median tooth $A$ was initially placed in an inappropriate position. It was extruded, with its PDL, by $1 \mathrm{~mm}$. In order to obtain significant simulation results, a plane strain thickness was defined for all the elements. Each tooth and its PDL were expanded by their mesiodistal crown width value, and the bone was stretched by $10 \mathrm{~mm}$. 
Table 2. Total lengths and maximum mesiodistal widths of five teeth (Scheid et al., 2012).

\begin{tabular}{cccccc}
\hline & $\begin{array}{c}\text { Central } \\
\text { incisor }\end{array}$ & $\begin{array}{c}\text { Lateral } \\
\text { incisor }\end{array}$ & Canine & $\begin{array}{c}\text { First } \\
\text { premolar }\end{array}$ & $\begin{array}{c}\text { Second } \\
\text { premolar }\end{array}$ \\
\hline $\begin{array}{c}\text { Total length } \\
(\mathrm{mm})\end{array}$ & 20.8 & 22.1 & 25.9 & 22.4 & 22.1 \\
$\begin{array}{c}\text { Maximum width } \\
(\mathrm{mm})\end{array}$ & 5.3 & 5.7 & 6.8 & 7.0 & 7.1 \\
\hline
\end{tabular}

The biological parts were modeled as linear elastic and isotropic materials, as shown in Table 3. Since the purpose of this work was to simulate orthodontic tooth displacement as a function of the strains within PDL, the teeth and the bone were considered as homogenous materials. Their properties were defined as mean material parameters (Marangalou et al., 2009). In addition, according to Vollmer's study, it was demonstrated that the nonlinear behavior of PDL does not affect the deformation distribution (Vollumer et al., 1999). Therefore, PDL was defined as a linear elastic material.

Table 3. Mechanical properties of biological parts (Marangalou et al., 2009).

\begin{tabular}{lccc}
\hline Parameters & Tooth & PDL & Bone \\
\hline Young's modulus (MPa) & 20000 & 0.68 & 2000 \\
Poisson's ratio & 0.15 & 0.49 & 0.3 \\
\hline
\end{tabular}

To simulate the contact between the PDL with the tooth root, and with the bone, tie interactions were used. No boundary condition was applied on the teeth and their PDLs. The external sides of the bone were completely fixed in the three models, as illustrated in Figure 1.

\section{Orthodontic tooth correction process using orthodontic appliance}

Some studies have suggested that the orthodontic tooth movement is controlled by the strains generated within PDL rather than deformations or stresses within the alveolar bone (Bourauel et al., 1999). In fact, when an orthodontic load is applied on the tooth crown, the PDL is deformed. According to its deformation $\varepsilon$, the bone remodeling rate $\dot{R}$ is determined as expressed in Equation (1) (Qian et al., 2008). Below a minimum threshold of $0.03 \%$ of deformation, the bone remodeling is not initiated. When the strain is higher than $0.03 \%$, the bone remodeling is triggered as it is described in Equation (1) (Marangalou et al., 2009).

$$
\dot{R}(\mathrm{~mm} / \text { day })=\left\{\begin{array}{ll}
0 & \text { if } \varepsilon<0.03 \% \\
0.153 \times \varepsilon-0.0046 & \text { if } 0.03 \%<\varepsilon<0.3 \% \\
0.0414 & \text { if } \varepsilon>0.3 \%
\end{array}\right\}
$$

Since the orthodontic tooth movement required in our study was a tooth intrusion, the wire was initially activated by applying a vertical displacement of $1 \mathrm{~mm}$ on the median bracket before it was tied on the tooth crown. This first stage made it possible to deform the wire, so it could deliver force on the tooth crown during the treatment period. The next stage described the tooth displacement resulting from the simulation of bone remodeling coupled to the wire deactivation for a period of 30 days. Doing so, an iterative procedure was performed as shown in Figure 2. Each 
iteration simulates one day of treatment, and it consists of two steps: in the first one, the wire is released, and so a force is transmitted to each tooth through the bracket. This step allowed the occurrence of the initial tooth displacement by deforming the PDL. In the second step, the maximal principal strains within PDL were extracted and evaluated at each node of the outer surface, which is in contact with the bone. In addition, the values of initial displacement of the inner PDL surface were used to define the direction of the tooth motion. Using bone remodeling law, expressed in Equation (1), the displacements of the PDL outer surface nodes were calculated and correlated with the initial displacement direction. Then, the positions of alveolar bone nodes were obtained depending on the PDL movement. This loop was iterated until the end of the chosen treatment period. At the beginning of each new iteration, all the elements were imported in their new position. Besides, the stress state of NiTi wire, the transformation strain, and the martensitic volume fraction were imported from the previous calculations as initial state.

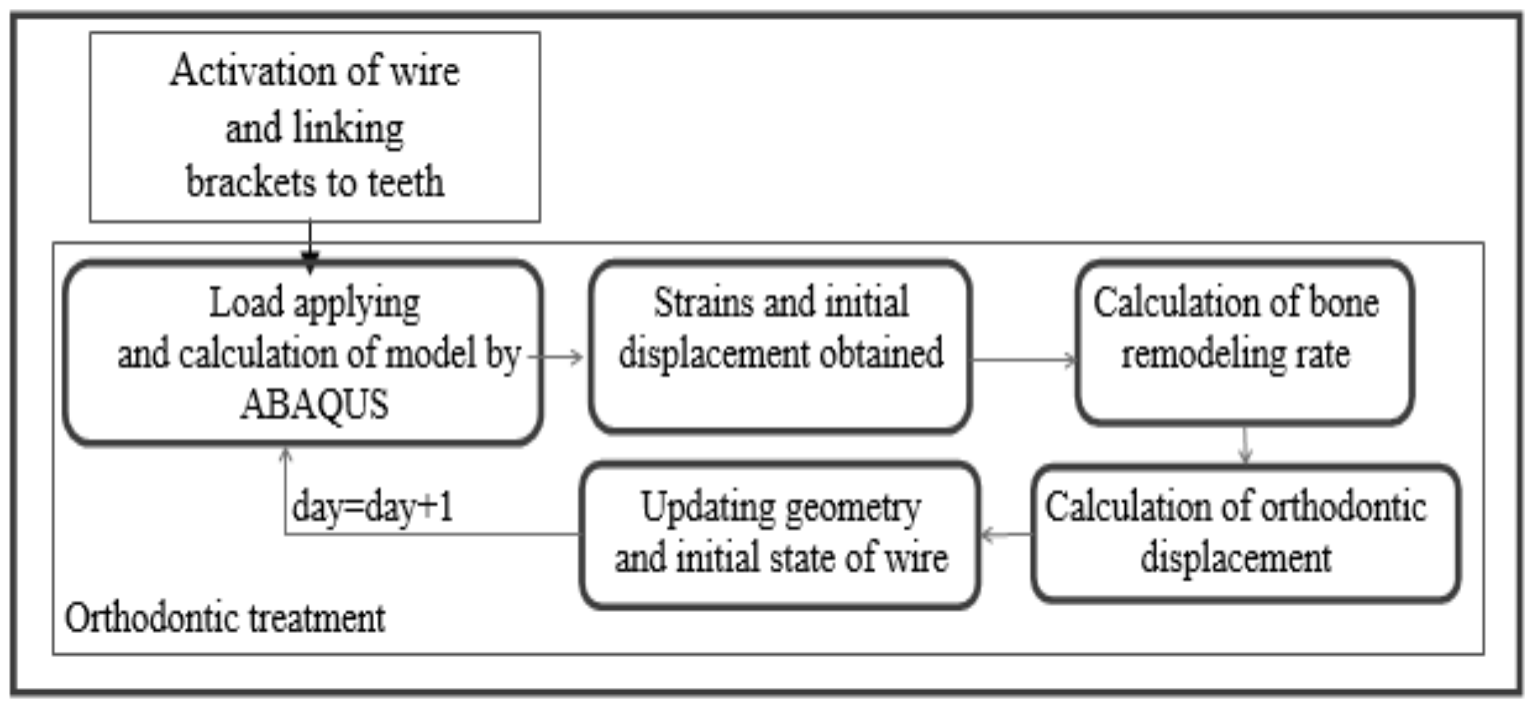

Figure 2. Long-term orthodontic tooth correction algorithm using NiTi superelastic arch wire.

\section{RESULTS AND DISCUSSION}

In order to simulate the most accurate orthodontic tooth correction using superelastic NiTi wire, we proposed an almost complete coupled biomechanical procedure. This procedure was tested on three two-dimensional models to reproduce an intrusion tooth movement that might be present at the beginning of the orthodontic tooth treatment. The novelty of this procedure consisted in activating the orthodontic wire to simulate its engagement in the oral cavity and then integrating the superelastic behavior of the NiTi alloy during long-term orthodontic tooth correction. This simulation allowed analyzing the effect of this wire behavior during the tooth alignment and leveling phase. In addition, a bone remodeling law was used to produce the teeth movement.

Figure 3 exhibits the load-deflection curves of the $\mathrm{NiTi}$ wire extracted from the reaction load obtained on the median bracket in the three models. The dash curves illustrate the activation load of the wire generated in the first stage. When deflection reaches $1 \mathrm{~mm}$, the action of the wire is equal to $12.28 \mathrm{~N}$. In the three models, the NiTi wire activation curve indicates that the bent region of the wire firstly undergoes an austenitic elastic deformation. When the wire is bent to almost $0.5 \mathrm{~mm}$, the change of the curve slope reveals the beginning of the martensitic transformation. The final loading state of the wire was considered as an initial one during the first day of treatment. During the orthodontic tooth correction, the brackets were bonded on the teeth crown, the wire was deactivated, and the developed algorithm, summarized in Figure 2, was executed. The solid curves present the load applied on the canine during the orthodontic tooth treatment. This load decreased gradually until the wire has resumed its original shape. 

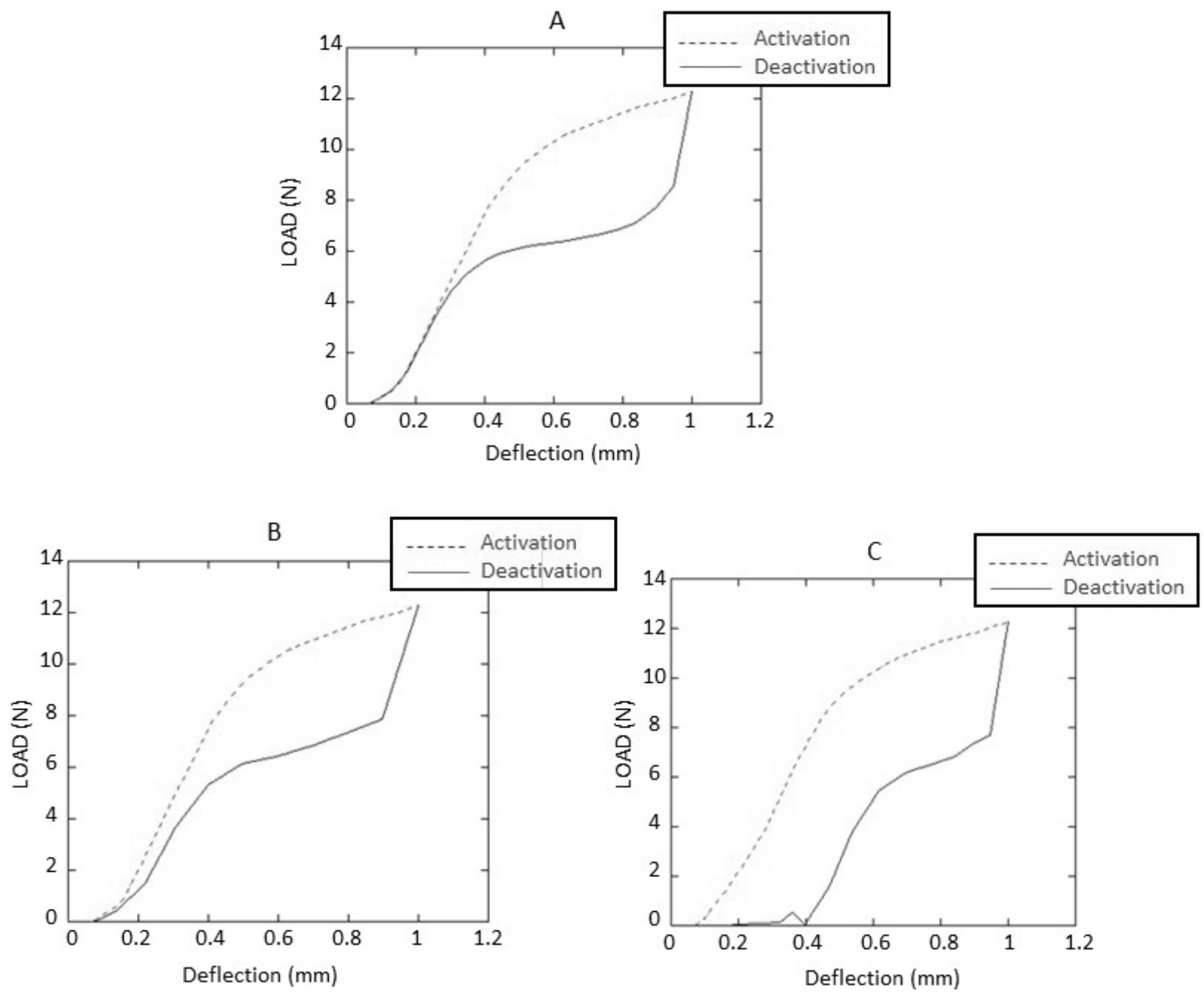

Figure 3. Load-deflection curves of three models: a) one-tooth model, b) three-teeth model, and c) five-teeth model.

The simulation of the orthodontic bone remodeling consisted of two steps. To produce the initial tooth displacement, the NiTi wire was unloaded. This step resulted in deforming the PDLs surrounding each tooth root in the three models. In all the models, PDL surrounding the canine was compressed and had the highest maximal principal strain value. When unloading the wire in the three and five teeth models, this wire acted also on the neighboring teeth, by applying different loads. Figure 4 illustrates the maximal principal strains within PDL for the second and third models during the first day of the orthodontic tooth treatment. The maximal tensile deformation values within PDLs surrounding the first premolar and lateral incisor were, respectively, $4.33 \%$ and $7.44 \%$ in the second model, and $4.06 \%$ and $7.08 \%$ in the third one. The maximal compressive strain, extracted from the PDL surrounding the canine, was $8.33 \%$ in the three-teeth model and $8.05 \%$ in the five-teeth model. Such deformation occurred at the root apex of the three teeth. Concerning the central incisor and the second premolar, the maximum strains were $1.8 \%$ and $1.08 \%$, respectively. They occurred at the mesial cervical and distal cervical margins. 


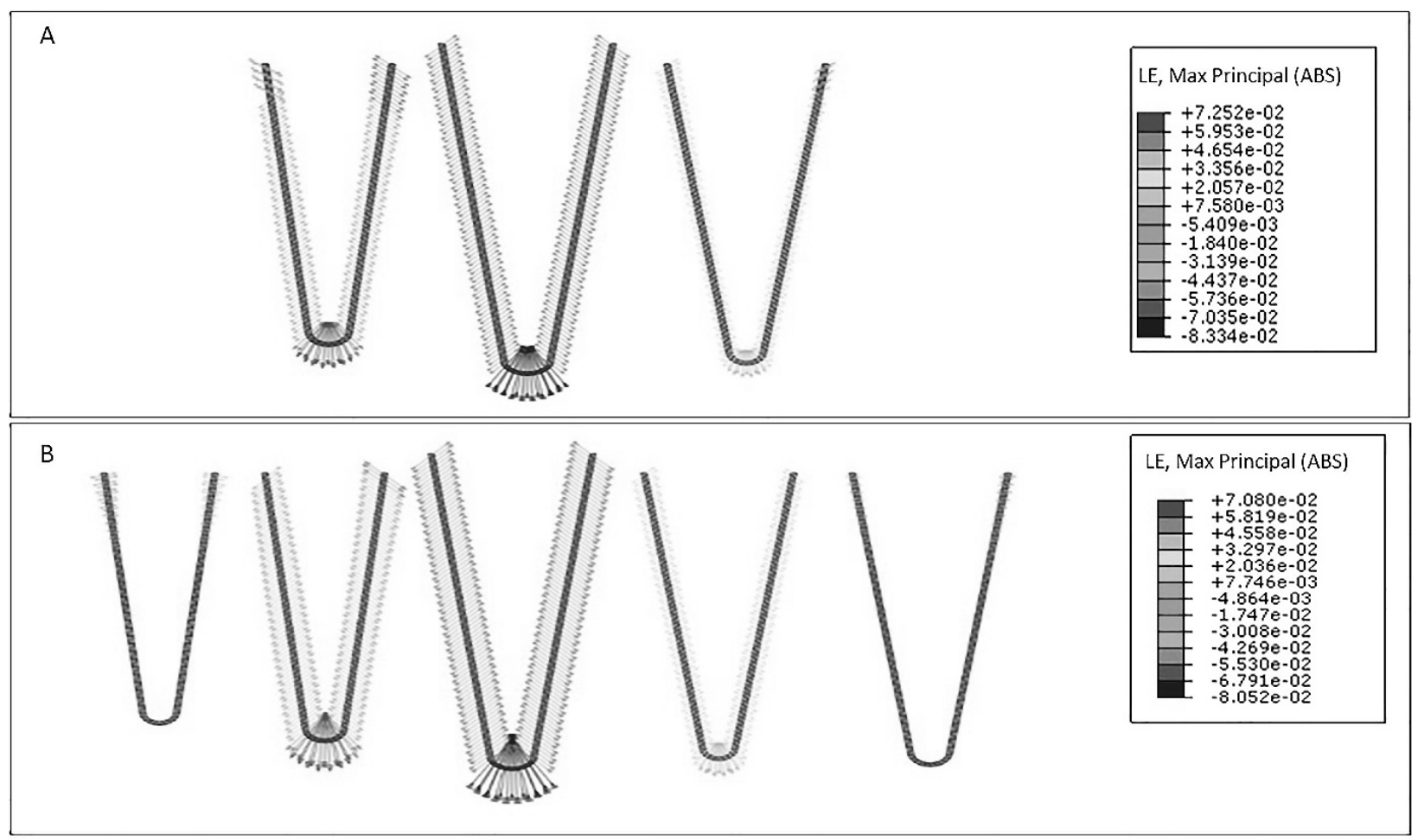

Figure 4. Maximal principal strain within PDLs of each tooth during initial displacement in first day of treatment: A) second model, B) third model.

The final teeth positions are depicted in Figures 5, 6, and 7. These illustrations exhibit the position of the teeth before and after the orthodontic tooth correction during the treatment period. In the first model, the canine was intruded by $0.92 \mathrm{~mm}$ after 30 days and the wire was able to almost recover its initial position. Since the left and right brackets were fixed, the displacement of the median one along the $Y$ axis, during the period of treatment, is presented in Figure 5. This displacement is proportional to the tooth movement. During the first 20 days, the tooth displacement increased, and then, it remained stable since the load was not high enough to stimulate the bone remodeling. The 10 last days, wherein the tooth did not move, might correspond to root resorption period that should have taken place at the beginning of treatment period. In fact, in the first day, the hydrostatic pressure average within the PDL surrounding the canine was equal to $0.19 \mathrm{MPa}$. This value was greater than the systolic blood pressure, and typically, root resorption should have been produced and blocked the tooth motion for ten days (Field et al., 2009). Besides, this model did not consider the presence of other teeth in the oral cavity. During wire deactivation and orthodontic tooth therapy, the presence of the plateau in the unloading curve, depicted in Figure 3(A), proved that the wire underwent backward martensitic transformation while the canine was moving. This simulation exhibited the capability of the wire to resume its initial form when considering a single tooth system in case the orthodontist utilizes the anchorage method to displace one desired tooth. 

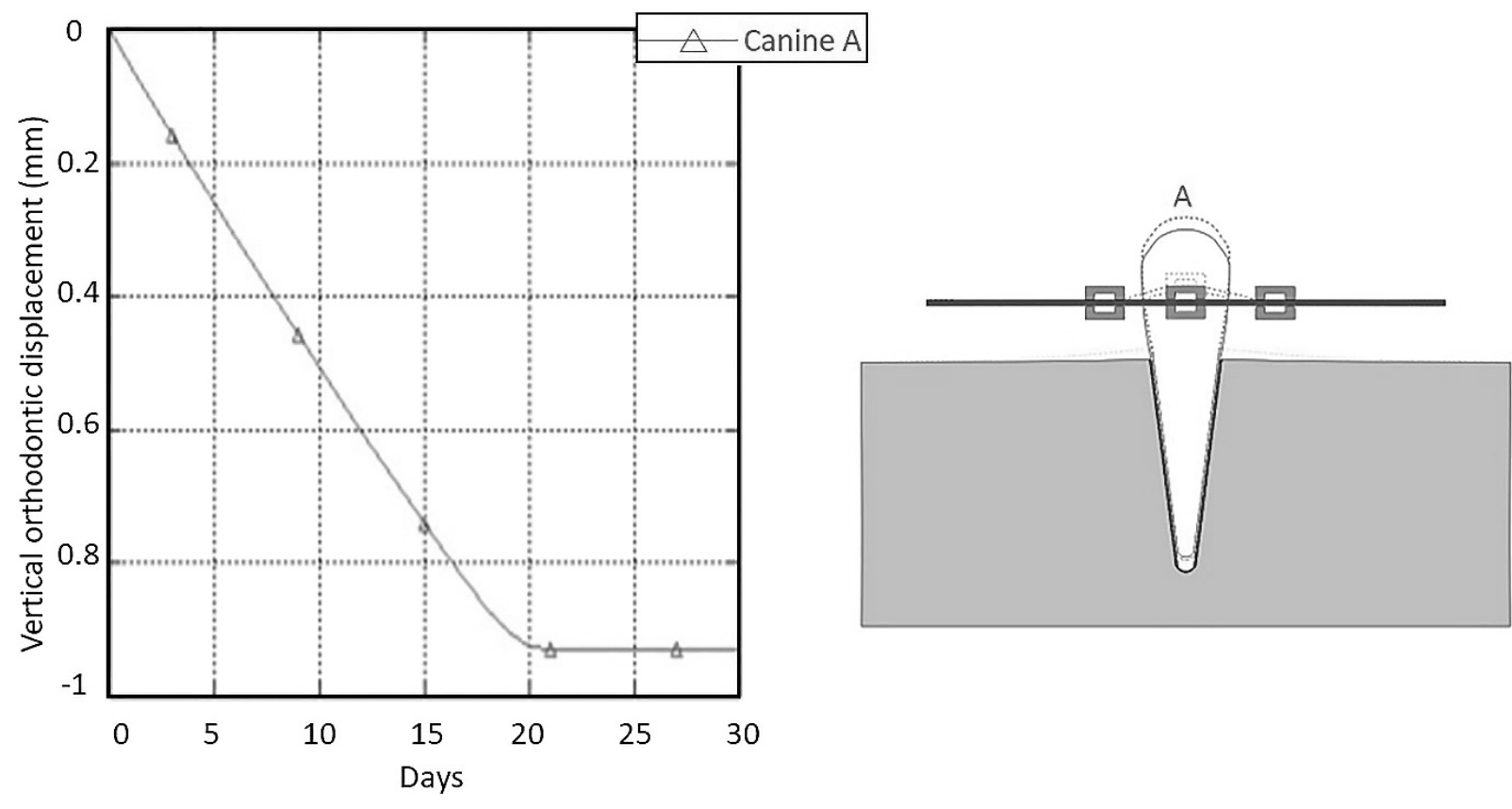

Figure 5. Orthodontic movement of canine in first model along treatment period.

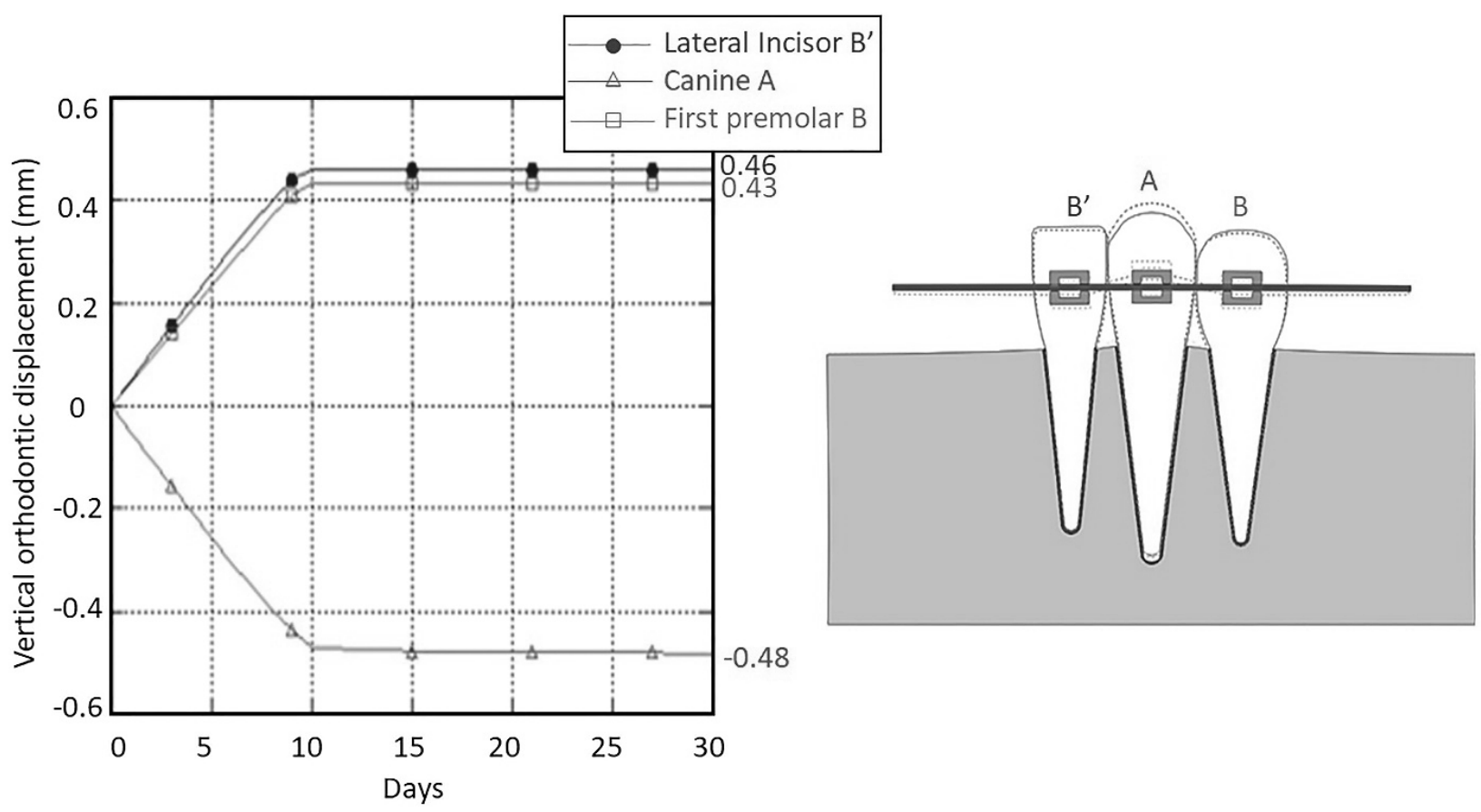

Figure 6. Orthodontic teeth movement of second model along treatment period. 


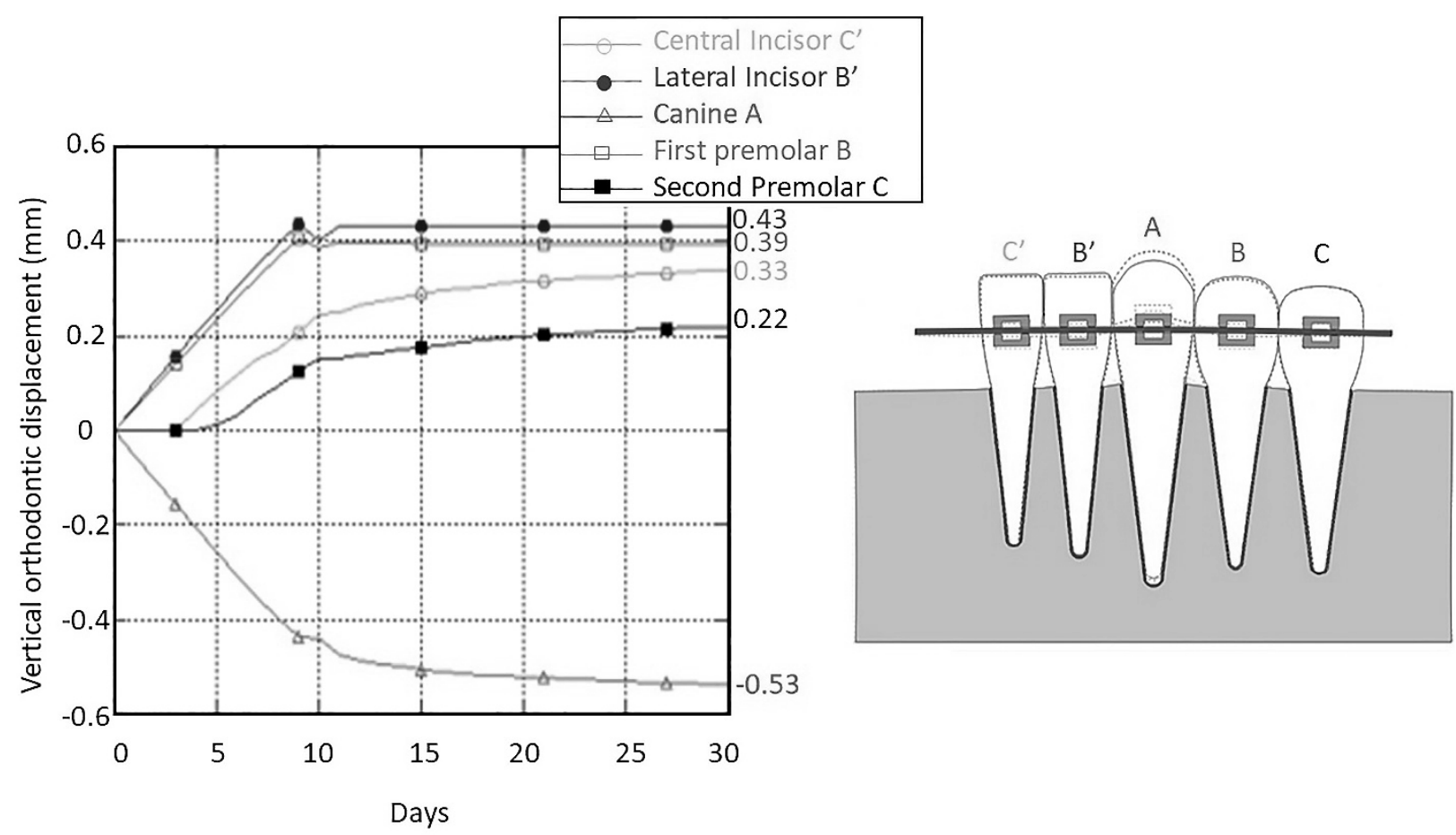

Figure 7. Orthodontic teeth movement of third model along treatment period.

Regarding the second model, the three teeth were able to move depending on strain within PDL as shown in Figure 6. This teeth alignment correction was achieved using the activated NiTi wire. As displayed in Figure 3(B), during unloading, the median region of the wire was submitted to backward transformation until it would completely become austenite. At the end of therapy period, the wire, which was initially bent to $1 \mathrm{~mm}$, was not deformed, whereas the canine vertical motion was about $0.5 \mathrm{~mm}$. In fact, the arch wire acted on the left and right brackets, which led to the teeth upward movement until aligning the wire. At the end of the treatment period, the canine was intruded by 0.48 $\mathrm{mm}$; the incisor and the premolar were extruded from the bone by 0.46 and $0.43 \mathrm{~mm}$, respectively. Besides being extruded, the teeth $B$ and $B$ ' were also displaced along the horizontal axis by 0.1 and $0.09 \mathrm{~mm}$, respectively. The upward motion of the left and right teeth was derived by activating the bone remodeling process during the first 10 days. After that, it stagnated during the rest of the chosen treatment period. Indeed, the load applied on those teeth was less than $0.035 \mathrm{~N}$, which did not permit triggering the bone remodeling mechanism, whereas the huge amount of the canine displacement was achieved during the first 10 day. After this period, this tooth continued to move with a slow velocity. As depicted in Figure 8, the maximal principal strain within the PDL surrounding the canine apex is still above the minimal threshold value that initiates the biological phenomenon responsible for tooth movement.

Figure 7 exhibits the motion result of the third model. The results of the three median teeth displacements were nearly similar to the second model results. Moreover, the two other teeth, which are the central incisor and the second premolar, were extruded by 0.33 and $0.22 \mathrm{~mm}$, respectively, and moved along the $X$ axis by about $0.1 \mathrm{~mm}$ in opposite directions. In addition, the vertical displacement of the three median teeth began from the first day of treatment, then, these teeth kept moving with a lower velocity compared to the past days, whereas the central incisor and the second premolar extrusion started from the fourth day and the fifth day, respectively. In the first 24 hours, they had only a slight reverse displacement along the $X$ axis and then stopped moving after 11 days. This result was in agreement with the explanation given by Faure (2011) who showed that for a first premolar extrusion movement, this tooth and its adjacent teeth would start moving at the first step. Then, the displacement of the other neighboring teeth would be 
activated in the next step. Indeed, during the first days of treatment, the wire did not apply a sufficient vertical load on the central incisor and the second premolar. The latter had a little degree of tipping caused by the frictional force generated in the orthodontic appliance contact zones. As depicted in Figure 4, the tipping motion resulted from the strain distribution along PDLs surrounding the central incisor and the second premolar. Next, when the three median teeth were displaced, the wire would be in a great contact with the bracket slots fixed on the central incisor and the second premolar crowns. Consequently, this would deform the PDLs surrounding the teeth, thus, the activation of the bone remodeling process in the vertical direction. Furthermore, during the first days, the load applied on the canine, the lateral incisor and the first premolar crowns was high enough to make them move with the maximum tooth movement velocity, which is about $0.35 \mathrm{~mm} /$ week. Figure 8 indicates that the strain within PDL surrounding the canine decreases in the $10^{\text {th }}$ day and increases in the $11^{\text {th }}$ day. The strain decrease resulted from the decline of the orthodontic load below the optimal force value, which is equal to $0.1 \mathrm{~N}$ (Proffit et al., 1999), because the wire was not enough in contact with the median bracket slot. During that day, the teeth $C^{\prime}$ and $C$ were extruded, and the teeth $B$ ' and $B$ were intruded, which led to align them. Consequently, in the $11^{\text {th }}$ day the new position of the wire permitted applying a sufficient load on the crown of tooth $A$, which caused the strain increase within the PDL. This result justified the fluctuation displayed in the deactivation curve illustrated in Figure 3(C). Afterwards, the lateral incisor and the first premolar stopped moving since the wire reaction on their bracket slots was not high enough to trigger the bone remodeling mechanism. Nevertheless, the canine, the central incisor and the second premolar were still displaced with a lower velocity. This decrease of teeth velocity resulted from the drop of the load generated from the wire during unloading. This decline affected the deformation within PDLs. As presented in Figure 8, after the $11^{\text {th }}$ day, the strain within PDL surrounding the canine kept declining, but it was still above the minimum threshold. At the end of therapy period, Figure 3(C) shows that the wire is not totally aligned, and a residual deflection is preserved. This was due to the opposite tipping of the teeth $C^{\prime}$ and $C$, which kept maintaining the curved shape of the wire.

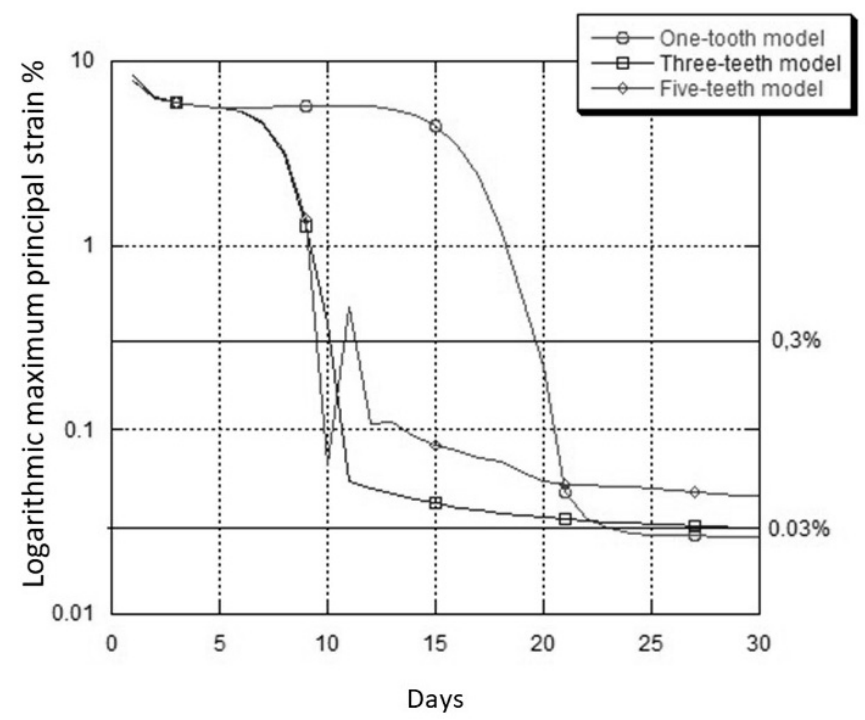

Figure 8. Absolute value of maximal principal strain obtained at PDL apex of canine along treatment period in three models displayed in logarithmic scale.

These results demonstrated that the amount of the orthodontic tooth displacement varied as a function of the considered teeth number. In fact, in the first model, as the two extreme brackets were fixed, the only targeted tooth was the canine. This tooth could be intruded until the complete alignment of the brackets, i.e., the canine was displaced by almost $1 \mathrm{~mm}$. This model did not consider the presence of the other teeth in the oral cavity when the wire was 
deactivated and reacted by-itself. This analysis demonstrated that for tooth intrusion movement, the numerical simulation had to take into account the effect of the neighboring teeth, at least the closed ones to the irregular tooth during the tooth alignment stage. The central incisor and the second premolar did not significantly affect the amount of displacement of the irregular tooth. However, the obtained results using one tooth were different from the results of the other models. According to Faure (2011), in order to study the displacement of an irregular tooth, the arch wire could be divided into unitary elements between the brackets, and only the reaction of the elements on the neighboring teeth was required.

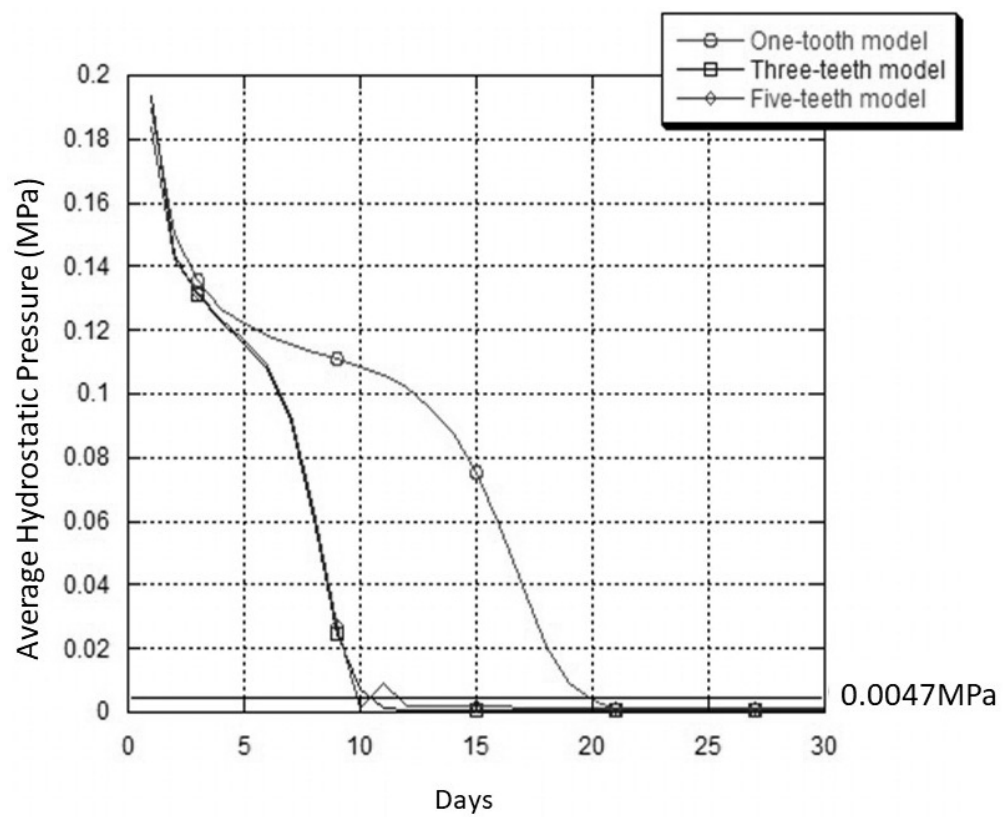

Figure 9. Average Hydrostatic Pressure during orthodontic treatment period in three models.

On the other hand, in order to evaluate the efficiency of the bone remodeling law adopted in our study, the evolution of the average pressure within the PDL surrounding the canine was evaluated and compared to the threshold value, which was adopted by Chen et al. (2014) in their work to initiate the orthodontic tooth movement. Figure 9 exhibits the average pressure variation during the orthodontic tooth correction. In the three models, the average hydrostatic stress was around $0.19 \mathrm{MPa}$ in the first day. This value was high enough to trigger the bone remodeling since it was greater than the threshold value, which is equal to $0.0047 \mathrm{MPa}$. Then, this pressure was declining as the orthodontic load was decreasing. In the first model, the hydrostatic pressure reached the threshold value in the $20^{\text {th }}$ day, whereas it attained the value of $0.0047 \mathrm{MPa}$ between the $10^{\text {th }}$ and $11^{\text {th }}$ day for the two other models. After those periods, according to the law adopted by Chen et al. (2014), the bone remodeling could not be triggered, and the tooth should not move. These assessments agree with the results provided by our simulation since the canine stopped moving from those same days as illustrated in the figures 5,6 and 7. Nevertheless, considering the pressure average within the PDL as a mechanical stimulus does not allow to reflect properly the distribution of the local hydrostatic stress along the PDL. As it is exhibited in Figure 10, in the three model during the first day, the pressure within the PDL apex is greater than the average pressure. While, this hydrostatic stress is closer to the averaged value in the cervical margins. 


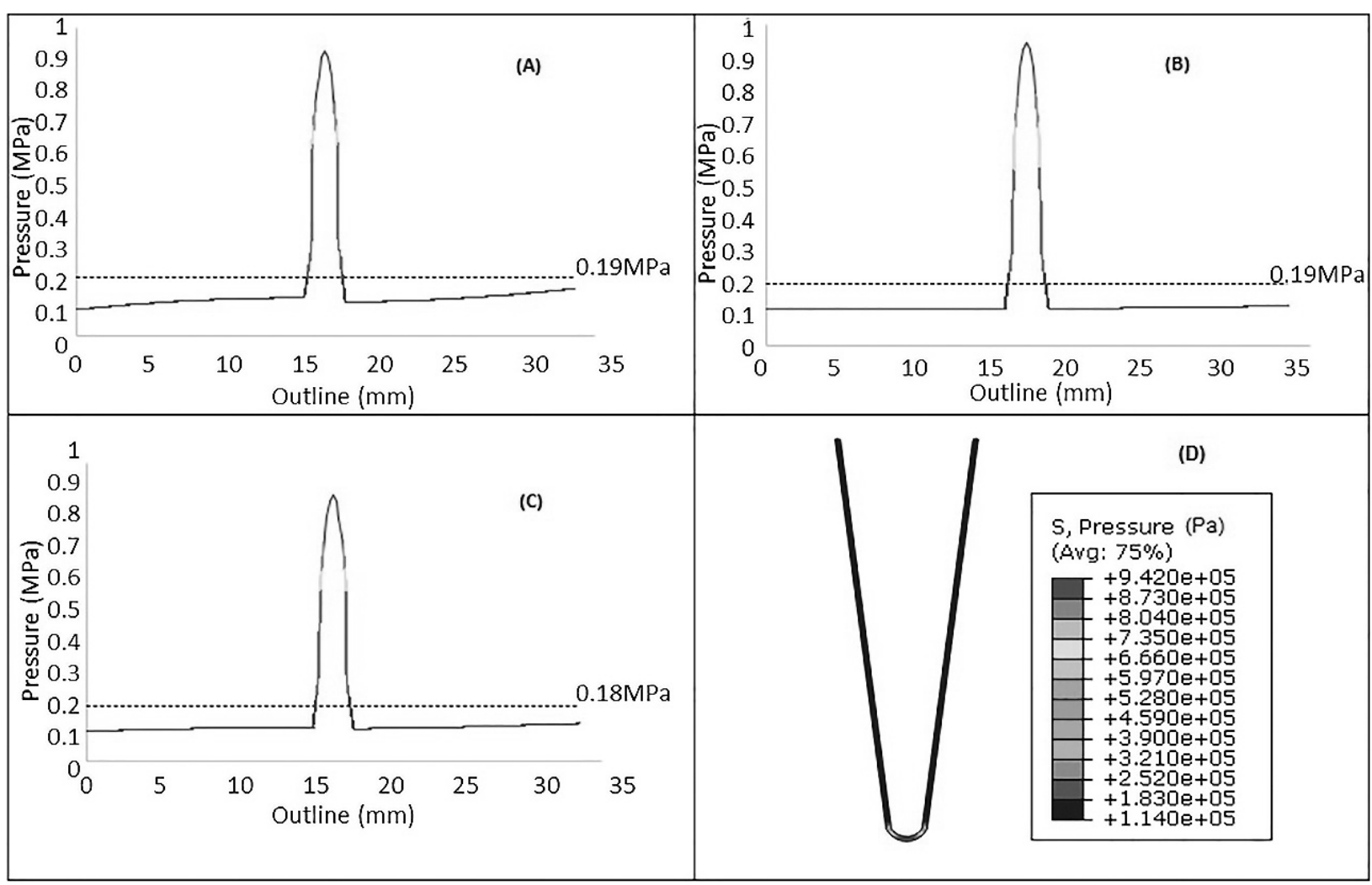

Figure 10. Pressure distribution along PDL surrounding canine outline during the first day of the treatment in : (A) the first model, (B), (D) the second model, and (C) the third model.

Thus, the developed method permitted to link the mechanical and biomechanical phenomena by coupling the orthodontic appliance action and the bone remodeling mechanism, which are responsible of producing respectively the initial tooth displacements and the orthodontic tooth movement. Moreover, utilizing an activated superelastic wire enabled perceiving the effect of its unloading in the oral cavity. In reality, when the orthodontist engages this wire in the brackets, the action of the arch wire will affect all the teeth and not only the irregular tooth. Therefore, this simulation permitted to evaluate the teeth motion rate and the estimate the maximal strain values that could be present within the PDLs.

Despite the fact that, this analysis combined the reaction of a superelastic wire and the bone remodeling process during a long-term therapy, it had some limitations. First, this simulation was two-dimensional. It gave a global idea of what is expected to produce in the oral cavity and allowed saving time calculation. Yet, the results showed that the inter-bracket distance and the teeth dimensions had an impact on the orthodontic tooth treatment, so it would be better to take into consideration the real geometry of the teeth and extract their three-dimensional shapes from computed tomography images. Then, the interaction between the teeth was not considered during this analysis. Finally, the developed algorithm did not assume that the root resorption could occur. In reality, when the load is higher than the optimal force required to stimulate the bone remodeling, the root resorption will damage the periodontium and obstruct the tooth movement during few days (Proffit et al., 1999) In further research, the aforesaid limitations should be taken into consideration and improved to simulate a three-dimensional orthodontic tooth correction based on the aforementioned procedure. 


\section{CONCLUSION}

In this study, a two-dimensional numerical orthodontic tooth correction was performed to produce a canine intrusion motion during the tooth alignment phase. This teeth alignment correction was achieved using a superelastic NiTi wire coupled to a bone remodeling process. The proposed procedure took into account the activation of the orthodontic wire to fit the brackets positions. Then, during a long-term teeth alignment, the declining force was generated from unloading the wire as it is supposed to occur in the oral cavity. The superelastic behavior of this wire was tested on one, three, and five teeth. As a result, the irregular tooth was intruded, and the left and right teeth were extruded as it is observed in the clinical experiences. In addition, the results permitted elucidating the response and effects of the orthodontic wire when it was engaged in the brackets during the tooth alignment stage. The strains within PDL, which were considered as the stimulus to produce the bone remodeling, declined as the load decreased during the treatment period. Moreover, hydrostatic pressure within PDL surrounding canine was investigated and compared to the blood pressure. It was determined that the root resorption could take place at the beginning of the treatment as it was mentioned in many studies. The results of this work provided a clear idea of the NiTi wire action and the teeth reaction in the oral cavity and could be used to predict the clinical teeth movement.

The purpose of our future work is to present a three-dimensional numerical simulation of a long-term orthodontic tooth displacement using the superelastic NiTi wire to simulate accurately different orthodontic tooth correction by considering the occurrence of the root resorption and study the three-dimensional biomechanical response of PDL, which is responsible of the bone remodeling.

\section{ACKNOWLEDGMENT}

The authors extend their appreciation to the Deanship of Scientific Research at King Khalid University, Saudi Arabia for funding this work through the Research Group Program under grant no. R.G.P.2/127/42.

\section{REFERENCES}

Aghili, H., Yasssaei, S., Ahmadabadi, M.N., \& Joshan, N. 2015. Load deflection characteristics of nickel titanium initial archwires. Journal of Dentistry, 12(9): 695-704.

Ben Naceur, I., Charfi, A., Bouraoui, T., \& Elleuch, K. 2014. Finite element modeling of superelastic nickel-titanium orthodontic wires. Journal of Biomechanics, 47(15): 3630-3638.

Bourauel, C., Freudenreich, D., Vollmer, D., Kobe, D., Drescher, D., \& Jäger, A. 1999. Simulation of orthodontic tooth movements. A comparison of numerical models. Journal of Orofacial Orthopedics, 60(2): 136-151.

Canales, C., Larson, M., Grauer, D., Sheats, R., Stevens, C., \& Ko, C.C. 2013. A novel biomechanical model assessing continuous orthodontic archwire activation. American Journal of Orthodontics and Dentofacial Orthopedics, 143(2): $281-290$.

Chen, J., Li, W., Swain, M.V., Darendeliler, M.Ali., Li, \& Q. 2014. A periodontal ligament driven remodeling algorithm for orthodontic tooth movement. Journal of Biomechanics 47: 1689-1695.

Elkhal Letaief, W., Fathallah, A., Hassine, T., \& Gamaoun, F. 2018. Finite element analysis of hydrogen effects on superelastic NiTi shape memory alloys: Orthodontic application. Journal of Intelligent Material Systems and Structures, 29: 3188-3198.

Elkhal Letaief, W., Hassine, T., \& Gamaoun, F. 2017. A coupled model between hydrogen diffusion and mechanical behavior of superelastic NiTi alloys. Smart Materials and Structures, 26(7): 11.

Faure, J. 2011. Biomécanique orthodontique. EDP Sciences, France.

Field, C., Ichim, I., Swain, M.V., Chan, E., Darendeliler, M.A., Li, W., \& Li, Q. 2009. Mechanical responses to orthodontic loading: A 3-dimensional finite element multi-tooth model. American Journal of Orthodontics and Dentofacial Orthopedics 135(2): 174-181.

Gannoun, M., Hellara, M.L., Bouby, C., Ben Zineb, T., \& Bouraoui, T. 2018. Numerical simulation of the force generated by a superelastic NiTi orthodontic archwire during tooth alignment phase: Comparison between different constitutive models. 
Materials Research Express, 5(4): 45405.

Hamanaka, R., Yamaoka, S., Anh, T.N., Tominaga, J.Y., Koga, Y., \& Yoshida, N. 2017. Numeric simulation model for longterm orthodontic tooth movement with contact boundary conditions using the finite element method. American Journal of Orthodontics and Dentofacial Orthopedics 152(5): 601-612.

Marangalou, J.H., Ghalichi, F., \& Mirzakouchaki, B. 2009. Numerical simulation of orthodontic bone remodeling. Orthodontic Waves 68(2): 64-71.

Mohd, J., Leary, M., Subic, A., \& Gibson, M.A. 2014. A review of shape memory alloy research, applications and opportunities. Materials and Design, 56: 1078-1113.

Moore, R.J., Watts, J.T.F., Hood, J.A.A., \& Burritt, D.J. 1999. Intra-oral temperature variation over 24 hours. European Journal of Orthodontics, 21(3): 249-261.

Proffit, W.R., Fields, H.W., \& Sarver, D.M. 1999. Biological Basis of Orthodontic Therapy. In: Proffit. W.R., Fields. H.W., \& Sarver. D.M. (3rd Ed.) Contemporary orthodontics. Pp. 296-325. Elsevier Mosby, Mosby.

Qian, Y., Fan, Y., Liu, Z., \& Zhang, M. 2008. Numerical simulation of tooth movement in a therapy period. Clinical Biomechanics, 23(suppl.1): 48-52.

Qidwai, M.A., \& Lagoudas, D.C. 2000. Numerical implementation of a shape memory alloy thermomechanical constitutive model using return mapping algorithms. International Journal For Numerical Methods In Engineering, 47(6): 1123-1168.

Razali, M.F., \& A.S. Mahmud. 2019. Computational Study on the Effect of Contact Friction towards Deactivation Force of Superelastic NiTi Arch Wire in a Bracket System. Materials Research Express, 6(8): 085709.

Scheid, RC. \& Weiss, G. 2012. Woelfel's dental anatomy, 8th edn. Lippincott Williams \& Wilkins, Philadelphia, USA

Vollmer, D., Bourauel, C., Maier, K., \& Jäger, A. 1999. Determination of the centre of resistance in an upper human canine and idealized tooth model. European Journal of Orthodontics, 21(6): 633-648.

Wagner, M.F., \& Schaefer, A. 2010. Macroscopic versus local strain rates during tensile testing of pseudoelastic NiTi. Scripta Materialia, 63(8): 863-866.

Wang, C., Han, J., Li, Q., Wang, L., \& Fan, Y. 2014. Simulation of bone remodelling in orthodontic treatment. Computer Methods in Biomechanics and Biomedical Engineering, 17(9): 1042-1050.

Zargham, A., Geramy, A., \& Rouhi, G. 2016. Evaluation of long-term orthodontic tooth movement considering bone remodeling process and in the presence of alveolar bone loss using finite element method. Orthodontic Wave, 75(4): 85-96. 